\title{
Anabases
}

ANABASES Traditions et réceptions de l'Antiquité

28 | 2018

Varia

\section{Une farce antique à la sauce moderne : comment traduire le théâtre de Plaute aujourd'hui ?}

Ancient Comedy with a Modern Twist: How Should We Translate Plautus' Drama today?

Clara Daniel

\section{OpenEdition}

\section{Journals}

Édition électronique

URL : http://journals.openedition.org/anabases/7618

DOI : 10.4000/anabases.7618

ISSN : 2256-9421

Éditeur

E.R.A.S.M.E.

Édition imprimée

Date de publication : 9 novembre 2018

Pagination : 111-125

ISSN : 1774-4296

Référence électronique

Clara Daniel, « Une farce antique à la sauce moderne : comment traduire le théâtre de Plaute aujourd'hui ? », Anabases [En ligne], 28 | 2018, mis en ligne le 09 novembre 2020, consulté le 20 janvier 2021. URL : http://journals.openedition.org/anabases/7618 ; DOI : https://doi.org/10.4000/anabases 7618

(c) Anabases 


\section{Une farce antique à la sauce moderne : comment traduire le théâtre de Plaute aujourd'hui ?}

Clara DANIEL

Y ou shall have a golden crown! Les fans de la série Game of Thrones reconnaîtront la savoureuse réplique de Daenerys lancée juste avant la mise à mort de son frère, condamné pour sa soif de pouvoir à recevoir sur le crâne de l'or brûlant issu d'une couronne fondue ${ }^{1}$. Les amateurs d'Histoire antique, quant à eux, y verront une réécriture divertissante de la sentence de Suréna, “ Rassasie-toi de ce métal dont tu es si avide! », général parthe qui tua Crassus Dives en lui versant dans la bouche de l'or fondu en allusion à sa cupidité proverbiale ${ }^{2}$. Voilà un exemple parmi tant d'autres d'une mode remarquable : la présence de références antiques dans la culture du divertissement contemporain ou culture pop. Des séries télévisées aux jeux vidéo en passant par la littérature jeunesse, les textes célèbres, événements historiques ou éléments mythiques - en un mot, la « culture antique »-sont devenus un vivier qui inspire et fait vendre ${ }^{3}$. Or, ce phénomène d'appropriation de l'antique fait naître un paradoxe moderne. Alors que d'un côté, un film comme Hunger Games a rapporté 155 millions de dollars en trois jours de projection aux États-Unis ${ }^{4}$, de l'autre, les lettres classiques sont en

1 D. Benioff et D. B. Weiss, sur une adaptation des romans de G. R. R. Martin, Game of Thrones, saison 1 épisode 6, 2011.

2 Dion Cassius, Histoire romaine XL, 27.

3 Cf. Antiquipop, le carnet scientifique tenu par F. Bièvre-PERrin qui recense « les références à l'Antiquité dans la culture populaire la plus contemporaine », http://antiquipop.hypotheses.org/a-propos, dernière consultation le 03/08/17.

4 Dans le premier volet de cette saga, le public a semblé apprécier ces gladiateurs de 
train de disparaître. Qui étudie aujourd'hui encore le grec ancien? Qui connaît les épisodes importants de l'Histoire romaine? Dans le système universitaire français, les spécialistes résistent mais le nombre d'étudiants dans les filières classiques devient alarmant ${ }^{5}$. Ainsi, alors que la culture antique, à travers des fragments proposés dans le divertissement contemporain, n’a jamais semblé aussi présente et démocratisée, la connaissance de l’Antiquité n’a jamais paru aussi minoritaire et en voie d'extinction.

Cette dichotomie radicale réactive une division plus ancienne entre lettres classiques destinées à une élite sociale et divertissement populaire de masses ${ }^{6}$. Mais puisque ces deux mondes peuvent toucher l'un et l'autre à la culture antique, comme nous l'avons évoqué avec la culture pop actuelle, un rapprochement n'est pas inenvisageable ${ }^{7}$. Afin de réfléchir à une manière possible de réconcilier ces deux domaines, nous allons nous intéresser au cas d'une victime collatérale dans ce divorce malheureux, le dramaturge latin Plaute. Pour expliquer son cas littéraire aujourd'hui, nous empruntons au raisonnement éclairant de V. Bessières, auteur d'une thèse portant sur la présence de l'Antiquité dans la postmodernité. D'après lui, la tendance européenne humaniste a procédé à une « réduction interne de l'Antiquité gréco-latine aux seules lettres classiques, à la seule République des Lettres $^{8}$. „ La conséquence de cette vision a été d’opposer grande littérature au

science-fiction combattant pour divertir l'élite d'une population opprimée par le gouvernement du Capitole.

5 Depuis 2011, le nombre d'admis au Capes de Lettres option lettres classiques a été inférieur au nombre de postes à pourvoir en raison d'un déficit de candidats.

6 Il faut nuancer doublement. D'une part, la division entre culture savante et culture populaire n'existe pas seulement dans le monde des lettres classiques. Il s'agit d'un phénomène plus global en art et en littérature que nous ne développerons pas ici. Pour une réflexion récente autour de la culture populaire, cf. H. N. PARKER, “ Towards a Definition of Popular Culture ", History and Theory, 50 2, (2011), p. 147-170. D'autre part, l'association entre lettres classiques et élite sociale ne va pas nécessairement de soi. Par exemple, ce n'est que depuis les années 1960 que l'enseignement du latin est socialement connoté. Sur le rapport entre éducation classique et élite socioéconomique, cf. É. Hall, “ Putting the Class into Classical Reception », in L. Hardwick et C. Stray (éd.), A Companion to Classical Reception, Malden, Blackwell, 2008, p. 386397.

7 Au Royaume-Uni, c'est le cas depuis une vingtaine d'années avec l'apparition des Classical Reception Studies qui ont commencé à percer également en France. Il s'agit d'une discipline tournée vers toutes les formes de réception de l'Antiquité, quels que soient le domaine culturel, l'aire géographique et la période concernés. Par conséquent, les séries télévisées ou le jeu vidéo se trouvent étudiés par des spécialistes de l'Antiquité au même titre que des objets plus traditionnels.

8 V. Bessières, “Le péplum, et après ? L'Antiquité dans les récits postmodernes », 
reste, c'est-à-dire la culture de masse : “[...] on aura tendance à opposer deux sortes de cultures, littéraires et spectaculaires, textuelles et orales, élitaires et populaires [...] » Or, dans le cas de Plaute précisément, cette réduction humaniste a déplacé la valeur de ses œuvres en les rangeant du côté des lettres : de ses comédies vivantes et populaires, interprétées par des marginaux de la société et destinées à un public hétéroclite ne nous est longtemps restée que l'idée de beaux textes littéraires. Depuis les années 60, des chercheurs comme F. Dupont ont contrebalancé cette image en mettant l'accent sur la notion de représentation ou performance ${ }^{9}$. "Le théâtre antique, partiellement confisqué par l'idéologie de l'Antiquité comme texte, c'était avant tout du spectacle $[\ldots]^{10}$. » Reste que, si cette conception est maintenant parfaitement normalisée dans les études plautiniennes, le dramaturge n'a toujours pas retrouvé sa valeur spectaculaire réelle en France. Il reste un auteur de bibliothèque, loué pour la pureté de son style grammatical ; ses textes, scrupuleusement traduits, décortiqués et commentés dans des éditions de spécialistes, sont, à la limite, montés en spectacle à l'ENS ou dans le cadre d'événements sur l'Antiquité ${ }^{\prime \prime}$. Mais en dehors d'un milieu universitaire, Plaute n'est jamais redevenu un classique à la mode pour le grand public. L'injustice qu'il subit est d'autant plus grande que ses héritiers se portent, eux, à merveille. En atteste outre-Atlantique le succès de la comédie musicale créée par S. Sondheim à Broadway en 1962, dont l'intrigue est un medley inventif de plusieurs scenarii de Plaute ${ }^{12}$. Héritier plus récent, plus lointain, mais tout aussi légitime : le sitcom américain, dont l'humour repose majoritairement sur le ressort du quiproquo, le public étant dans la confidence. Lorsque, dans un épisode de Friends, la sœur jumelle de Phoebe prend sa place pour jouer des tours à ses proches, les créateurs

Anabases, 16, 2012, mis en ligne le 01 octobre 2015, http://anabases.revues.org/3993, dernière consultation le 04.08.17. Il précise bien le fait que ce phénomène de catégorisation tient son origine dans l'Antiquité même.

9 F. Dupont, L'orateur sans visage : essai sur l'acteur romain et son masque, Paris, Presses universitaires de France, 2000 ; L'acteur-roi : le théâtre dans la Rome antique, Paris, les Belles Lettres, 2003. Voir également la préface à ses deux traductions de Plaute : La marmite, suivi de Pseudolus, Arles, Actes sud, 2001. Côté anglo-saxon, l'ouvrage majeur consacré à cette idée de spectacle reste celui de T. Moore, The theater of Plautus : Playing to the Audience, Austin, University of Texas Press, 1998.

V. Bessières, “ Le péplum, et après? ».

11 En 2014, dans le cadre de la journée “ Découvrir l'Antiquité » de l'École Normale Supérieure de Paris, H. Ollivier et L. Nagot ont monté, à partir d'anciennes traductions, une représentation du Soldat fanfaron. Lors des Dionysies 2016, N. Lakshmanan a présenté sa création personnelle d'Amphitryon.

12 S. Sondheim (musique), B. Shevelove et L. Gelbart (livret), d'après Plaute (Pseudolus, Miles gloriosus et Mostellaria), A Funny Thing Happened on the Way to the Forum, 1962. 
ne se doutent peut-être pas de l'hommage rendu à Plaute et ses intrigues reposant sur le personnage du double, comme dans Amphitryon ou les Ménechmes ${ }^{13}$.

Alors si d'une part, dans le monde académique, Plaute n'est plus seulement reconnu pour ses caractéristiques grammaticales et littéraires, mais bien pour la force de son jeu théâtral, si d'autre part, la valeur comique qu'il incarne semble avoir survécu dans la culture occidentale populaire, pourquoi ses textes sont-ils si peu joués à notre époque? Lex parsimoniae: si ses textes sont si peu joués, c'est peut-être avant tout qu'ils sont si peu jouables. Or, soulever cette notion de jouabilité, ou performabilité, de Plaute revient à s'interroger moins sur le texte latin que sur sa version française, et donc sur l'acte même de traduction. En effet, même si plusieurs facteurs peuvent être évoqués pour justifier la faible présence de la comédie latine sur scène ${ }^{14}$, nous nous concentrerons ici exclusivement sur la problématique du texte traduit. Plaute, par l'intermédiaire de ses différentes traductions, est-il aujourd'hui représentable sur scène à destination du grand public? Si nous prenons le texte français de référence au $x x^{\mathrm{e}}$ siècle, c'est-à-dire les traductions d'Alfred Ernout aux Belles Lettres, leur mise en scène semble aujourd'hui difficile ${ }^{15}$. La traduction, littérale et littéraire, n'est pas sans mérite pour la lecture et l'étude de Plaute. Mais le registre soutenu, le vocabulaire vieilli, le rendu fidèle des plaisanteries et des références culturelles font de ces textes des scripts théâtraux impraticables sans modification. Qui, sans être spécialiste d'Histoire romaine, peut savourer une plaisanterie faisant allusion à l'incarcération du poète Naevius? Ou apprécier le charabia punique de Romains déguisés en Carthaginois ${ }^{16}$ ? Plaisanteries et références qui sont d'ailleurs souvent explicitées en notes par le traducteur. Il s'agit en effet essentiellement d'une traduction pour la page: plaisir savant pour qui a le loisir de relire un passage, chercher sa signification, comparer avec le latin d'origine. Mais pour la scène, nous militons pour une autre traduction de Plaute, ni littérale ni (seulement) littéraire : un programme d'adaptations contemporaines qui, tout

13 M. Kauffman et D. Crane, Friends, saison 1 épisode 16, 1995. Dans la pièce des Ménechmes, le ressort comique est similaire : un jumeau arrive, semant le trouble dans l'entourage de son frère.

14 Ce n'est pas l'objet du présent article, mais il serait nécessaire de comparer avec l'omniprésence de la tragédie grecque sur les scènes occidentales.

15 A. Ennout (éd. et trad.), Plaute, Comédies, en 7 vol., Paris, Les Belles Lettres, rééd. 2003, première édition 1932-1940.

16 Pour la mention de l'emprisonnement du poète Naevius, contemporain de Plaute, cf. Miles gloriosus, v. 209-212. Dans une scène majeure du Poenulus, pièce qui a pour personnage principal un Carthaginois, un esclave romain tente d'imiter la langue punique (v. 975-1031). 
en restant le plus fidèle possible aux textes, puisse rendre accessible au grand public leur dimension comique et populaire. Dans la lignée de F. Dupont, et à la suite d'autres initiatives récentes ${ }^{17}$, nous défendons cette nécessité de retraduire Plaute afin qu'il soit à nouveau joué. À partir d'un extrait du Miles gloriosus, nous exposerons les différents principes qui président à notre vision. Puis, en revenant sur la problématique de l'éthique du traducteur et la question de la fidélité, nous défendrons la légitimité d'une transposition moderne en nous référant à la notion plautinienne de ludus.

\section{Du Soldat fanfaron au militaire Kislapet: moderniser le théâtre de Plaute}

Dans son article intitulé “Comment traduire Plaute? » lors d'un colloque du CRATA consacré au Miles Gloriosus, J.-P. Mazières envisage la problématique générale de la traduction des textes antiques : il y voit une « lutte, jamais achevée, pour réduire, autant que faire se peut, ce que l'on pourrait appeler le 'déficit' de sens - et de sensation(s) - par rapport à l'original ${ }^{18}$ ». La distance temporelle, géographique, littéraire et culturelle qui nous sépare de Plaute et, en même temps, l'impératif contradictoire de le traduire aujourd'hui fait qu'il en va du devoir du traducteur d'établir une stratégie personnelle pour résoudre la question de ce déficit de sens. La stratégie que nous défendons ici réside alors dans la modernisation de ces comédies anciennes. Nous prendrons comme exemple le Miles gloriosus, devenu dans la tradition littéraire française Le soldat fanfaron. Le premier geste de traduction qui nous semble s'imposer pour moderniser le texte est celui de la modification du titre. En effet, conserver l'adjectif « fanfaron », qui est peu usité - osons même, démodé - et relève d'un registre soutenu, ne paraît pas cohérent avec une stratégie d'adaptation contemporaine. Nous proposons, en titre programmatique, Le militaire Kislapet : en passant par l'expression familière “ se la péter », avec la transcription phonétique qui rappelle le langage sms, cela permet, tout en conservant le sens exact du latin gloriosus, d'actualiser cette pièce classique afin d'en imaginer une représentation moderne auprès du grand public. Le cœur de cible que nous visons est le public adolescent : car, au-delà de

17 Cf. J.-P. Mazıères, Le soldat fanfaron de Plaute : version versifiée, Toulouse, Le Mirail (CRATA), 1993. Le Groupe de Théâtre Antique de Neuchâtel, une collaboration entre des étudiants de l'université et le metteur en scène Guy Delafontaine, travaille également à partir d'une traduction actualisée des pièces antiques. Ils ont monté deux comédies de Plaute :Les Ménechmes : les Germains en 1993 et Rudens : les naufragés en 2013.

18 J.-P. MAzières, “ Comment traduire Plaute? », in CRATA, Autour du « Miles gloriosus » de Plaute : actes du colloque, Toulouse, Presses universitaires du Mirail, 1993, p. 81-90, p. 83. 
l'importance de la mise en scène, nous pensons qu'une traduction modernisée pourrait aussi servir dans un cadre scolaire à la pratique d'une lecture plus ludique des textes anciens, dont la découverte au collège ou au lycée peut ennuyer voire rebuter.

À partir de l'extrait ci-dessous et de la traduction que nous en proposons, nous allons réfléchir aux moyens de moderniser la pièce. Dans ce passage (v. 690700), le personnage de Periplecomenes, décrit par Plaute comme un « vieillard sympathique » (senex lepidus) et éternel célibataire, célèbre son refus de se marier en imitant le discours d'une épouse dépensière :

\begin{tabular}{|c|c|}
\hline $\begin{array}{l}\text { PERIPLECOMENES } \\
\text { [...] verum priusquam galli cantent, quae me somno suscitet, } \\
\text { dicat : Da mihi, vir, calendis meam quod matrem venetit; } \\
\text { da qui faciam condimenta, da quod dem quinquatribus } \\
\text { praecantrici, coniectrici, hariolae atque haruspicae; } \\
\text { flagitium'st, si nihil mittetur; quo supercilio specit! } \\
\text { Tum plicatricem, clementer non potest, quin munerem. } \\
\text { Iampridem, quia nihil abstulerit, subcenset ceriaria. } \\
\text { Tum obstetrix expostulavit mecum, parum missum sibi. } \\
\text { Quid ! nutrici non missurus quidquam, quae vernas alit?' } \\
\text { hae atque horum similia alia damna multa mulierum } \\
\text { me uxore prohibent, mihi quae huius simileis sermones serat. }\end{array}$ & $\begin{array}{l}\text { ROULEPATIN } \\
\text { Non, elle, avant même la sonnerie } \\
\text { du réveil, elle me tirerait du } \\
\text { sommeil pour me dire : “ Mec, file- } \\
\text { moi de quoi acheter un cadeau à } \\
\text { ma mère pour son anniversaire, } \\
\text { de quoi faire à bouffer, file-moi } \\
\text { de quoi donner pour Noël à ma } \\
\text { voyante, ma psy, ma coach et ma } \\
\text { prof de yoga. Ça ferait scandale si } \\
\text { je payais pas l'esthéticienne qui } \\
\text { m'a fait les sourcils! Et la femme } \\
\text { de ménage? C'est pas sympa de } \\
\text { pas lui verser de pourboire. Ça } \\
\text { fait un moment qu'elle se plaint } \\
\text { de pas avoir reçu son salaire, } \\
\text { la cuisinière. Et la sage-femme } \\
\text { est venue râler d'avoir pas été } \\
\text { assez payée. Ah et la baby-sitter } \\
\text { qui s'occupe des gosses? On lui } \\
\text { donnerait pas un p'tit quelque } \\
\text { chose?» Ces réclamations, ou } \\
\text { mille autres qu'ont les meufs pour } \\
\text { vous taxer, m'empêchent d'en } \\
\text { épouser une qui me sorte la même } \\
\text { rengaine. }\end{array}$ \\
\hline
\end{tabular}

“ À travers la langue travaillée de Plaute, travaillée parce qu'elle est versifiée et créative, les Modernes s'accordent à déceler une langue familière $[. . .]^{19}$. » Là réside toute la tension interne à l'œuvre théâtrale de Plaute qui place ses textes à la fois dans la grande littérature et le divertissement populaire. Nous nous trouvons face

19 F. GAIDE, “ A propos des interactions verbales dans le théâtre de Plaute » in C. Moussy (éd.), De lingua latina nouae quaestiones, Actes du Xe Colloque International de Linguistique latine, Paris-Sèvres, 19-23 avril 1999, Louvain, Paris, Peeters, 2001, p. 959969, p. 964. 
à un travail d'écriture poétique qui vise in fine à reproduire les codes de l'oral : via un caractère artificiellement familier et improvisé, la parole plautinienne transporte le spectateur dans une conversation réaliste qui a lieu à Rome au ${ }^{\mathrm{III}^{\mathrm{e}}}$ siècle av. J.-C. ${ }^{20}$. Sans oublier les procédés littéraires et poétiques qui soustendent le texte du Miles gloriosus, nous avons donc visé dans cet exemple à un résultat semblable en surface pour un spectateur contemporain : une conversation quotidienne réaliste transposée au xxi ${ }^{\mathrm{e}}$ siècle. Mais comment rendre en français cette familiarité du style oral ? En gardant en tête notre double objectif (la scène et une cible jeune), nous avons procédé à des choix stratégiques qui peuvent faire débat. En premier lieu, nous avons opté pour la prose. En effet, nous avons estimé que la complexité des vers de Plaute (qui alterne entre diuerbium, le vers parlé, et cantica, qui comporte effets lyriques et chansons) et leur effet produit seraient mal transposés par des vers français. Que ce soit avec l'alexandrin ou le décasyllabe, la dimension littéraire instaure un registre soutenu qui, avons-nous jugé, aurait diminué l'effet de conversation familière que nous recherchions. En revanche, le fait de ne pas reproduire la versification n'empêche pas de prêter attention à la musique du texte d'origine : l'ordre syntaxique, l'attention portée à la quantité de syllabes ou de mots, le tempo, les accents, les effets sonores de type allitératif, toutes ces questions de rythme qui débordent la seule versification, n'ont pas été éludées. En second lieu, nous avons favorisé l'hypocorrection syntaxique. L'idée était d'utiliser la grammaire de la langue orale informelle : mode interrogatif simple, suppression de l'adverbe négatif “ ne » ( On lui donnerait pas...? »), préférence pour le style paratactique et refus d'enchaîner des coordinations ou subordinations complexes (cf. la relative quae du premier vers transformée en proposition principale). Nous avons également favorisé les procédés qui reflètent la prononciation orale comme les interjections familières (le quid traduit par « ah et » plutôt que par une exclamation plus formelle), les apocopes (« sympa ») et les élisions et autres modifications orthographiques (“ ça », “ p’tit ). Enfin, la familiarité de la structure syntaxique ne pouvait pas tenir sans une adaptation cohérente du lexique, qui combine les registres courant et familier. Nous avons particulièrement favorisé les emprunts au parler jeune (“meufs », “taxer»): via des éléments langagiers ponctuels, cette coloration du texte permet de faire signe en direction de la cible choisie pour cette adaptation, les adolescents, en créant les conditions

20 Sur ces deux niveaux d'écriture, cf. F. Dupont, « L'écriture théâtrale antique : le dialogue théâtral, échanges ritualisés et conversations », in J. Lallot et D. Peтiт, Lalies, 20 (1999), Paris, Éditions Rue d’Ulm, 2001, p. 145-150. Sur les procédés d'oralité chez Plaute, cf. G. E. Duckworth et R. L. Hunter (éd.), The Nature of Roman Comedy: a Study in Popular Entertainment, Norman, University of Oklahoma Press, 1994, chap. " Language and Style » p. 332-340. 
d'une complicité potentielle ${ }^{21}$. Cependant, à l'échelle de toute la pièce, le choix fait en amont d'inscrire le texte dans certains registres (courant et familier) pose la question de l'uniformité langagière : s'agissant d'une pièce de théâtre, ce partipris ne tendrait-il pas à lisser la diversité, qui, dans le texte original, caractérise la parole des différents personnages? S'il s'agit incontestablement d'une objection légitime, une observation plus fine de la langue plautinienne permet d'en limiter la pertinence. Car, même si le langage joue chez Plaute un rôle déterminant dans la caractérisation des personnages, celle-ci ne passe pas nécessairement par la problématique du registre de langue. Ainsi, C. Filoche, dont la thèse, fondée sur des relevés statistiques minutieux, établit plusieurs éléments caractéristiques du langage des personnages plautiniens, n'évoque jamais la question de leur niveau de langue comme un possible critère discriminant ${ }^{22}$. L'auteur met au contraire en valeur d'autres critères de différenciation, comme les images littéraires, les champs lexicaux ou les figures de sonorité. Nous pouvons donc imaginer une traduction qui concilierait sans contradiction homogénéité du niveau de langue et hétérogénéité dans les répliques. Au-delà de ces questions de style, si l'on veut donner au texte une facture contemporaine et familière, apparaît un problème complémentaire, celui des références.

En traductologie, les realia désignent le contenu réaliste de l'univers dans lequel un texte a été écrit. Dans le cas de Plaute, il s'agit donc de tous les éléments de la “culture antique » et, plus précisément, d'une situation bilingue complexe qui englobe à la fois des références précises à la société romaine contemporaine de l'auteur et des références grecques dues au modèle original de la pièce. Or, dans le cas d'une traduction qui, se voulant la plus fidèle possible, reste littérale, le décalage entre Antiquité et modernité va empêcher un public qui n'est pas spécialiste d'apprécier hic et nunc une allusion politique, une référence

21 Dans le cadre de cet article, nous ne pouvons entrer dans le détail de la complexité sociolinguistique des registres de langue. Pour un aperçu général, voir par exemple les travaux de F. Gadet, comme “ Niveaux de langue et variation intrinsèque », Palimpsestes, 10, 1996, mis en ligne le 30 septembre 2013, http://palimpsestes.revues.org/1504, dernière consultation le 12.03.18. Sur les théories du " parler jeune », cf. M. Auzanneau et C. JuILlard, “ Introduction. Jeunes et parlers jeunes : catégories et catégorisations », Langage et société, 141, (2012/3), p. 5-20.

22 C. Filoche, La caractérisation par le langage des personnages de la comédie plautinienne, sous la dir. de J. C. Dumont, Université Paris Nanterre, thèse soutenue le 16 octobre 2004. Voir également la thèse de M. E. Gilleland, Linguistic Differentiation of Character Type and Sex in the Comedies of Plautus and Terence, Université de Virginie, Charlottesville, 1979. Si existent à présent plusieurs études stylistiques sur la langue de Plaute, la question d'une possible différenciation des personnages par leur niveau de langue reste en revanche à explorer. 
littéraire, ou un stéréotype ethnique. Afin d'éviter ce déficit de sens dont parle J.-P. Mazières, nous proposons ainsi d'adapter les realia du Miles gloriosus, c'està-dire de passer par des équivalents culturels actuels. L'extrait que nous avons choisi comprend un lexique technique spécifique concernant les pratiques religieuses romaines. Or, dans une adaptation, le but d'un équivalent est de créer à partir d'un autre référent culturel une réalité similaire dans la situation du dialogue. Par exemple, les Quinquatries (v. 3 dans l'extrait) désignent une fête religieuse consacrée à Minerve. Mais ici, le personnage utilise cette référence par rapport à un élément précis : les étrennes qu'on était censé dispenser à cette période. Le fait de transposer les Quinquatries à la fête de Noël fonctionne donc dans ce passage en ce que les deux fêtes représentent une célébration religieuse et culturelle, connue pour les présents que l'on offre. Si les Quinquatries avaient été mentionnées en rapport avec la déesse Minerve, l'utilisation de Noël n'aurait peutêtre pas fonctionné. Un équivalent est une adaptation située en contexte, contexte à la fois linguistique et culturel. De la même façon, l'accumulation des métiers de la voyance, qui conduit jusqu'à l'effet de ridicule (la voyante qui lit dans les sourcils v. 5), n'est pas neutre dans cette tirade comique fondée sur un stéréotype misogyne, celui de la dépensière. Periplecomenes dénonce ici des professions inutiles, voire des charlatans, qui profiteraient d'une clientèle féminine crédule. Nous avons essayé de reproduire la même gradation, en utilisant des métiers qui, selon un stéréotype actuel équivalent, représentent un coût élevé, visent plutôt les femmes, et pourraient être jugés par le personnage masculin comme sources de dépenses inutiles. La question de l'adaptation de Plaute est d'autant plus délicate que les références de ses pièces se décomposent en réalité en deux univers, à cause de la construction interne de son œuvre, qui repose sur un bilinguisme grécolatin. Nous n'allons pas développer ici la question complexe de ce bilinguisme plautinien $^{23}$, mais dans notre extrait, nous avons tenté de reproduire des effets ponctuels de langue étrangère (les termes et références grecs insérés dans un texte latin) en nous servant de l'univers anglo-saxon. Ainsi, nous utilisons dans notre traduction des emprunts linguistiques et culturels (“ coach », “ baby-sitter »). Dans un autre passage de la pièce, la mention de l'Iliade pourrait par exemple être transposée à celle de la saga américaine Game of Thrones ${ }^{24}$. Cela permettrait de jouer avec un décalage similaire, entre proximité et distance, de deux cultures perçues à la fois comme rivales et comme modèles (la culture grecque pour Rome, la culture américaine pour la France). La création d'une sorte d'imaginaire américanisé dans l'adaptation française du Miles instaurerait l'équivalent d'une

23 Cf. F. Dupont, “ Plaute, 'fils du bouffeur de bouillie' », in E. Valette-Cagnac et F. Dupont (éd.), Façons de parler grec à Rome, Paris, Belin, 2005, p. 175-209.

24 Miles gloriosus, v. 743 : east odiorum Ilias. 
grécité voulue par Plaute dans le jeu de sa propre adaptation latine ${ }^{25}$. Pour finir, il faut noter qu'une stratégie de l'équivalence a ses limites : puisqu'elle fonctionne à partir de l'apriori de l'existence d'éléments comparables dans deux cultures différentes, elle restera impuissante face à des éléments perçus comme uniques. C'est pourquoi dans le cas de Plaute, l'élément le plus difficile à traiter dans une traduction contemporaine sera celui du système de l'esclavage comme phénomène social spécifique. Il est possible, selon les dialogues, de transposer au monde du travail : le dominus deviendrait le patron, les autres serui les collègues de travail, mais c'est une équivalence très limitée qui ne se situe pas dans le même système de société. Un déplacement tel que celui-là conduit alors à soulever la question de la fidélité à Plaute. Si une adaptation contemporaine comme celle que nous proposons change les éléments culturels et utilise un langage familier, déplaçant la pièce à un imaginaire occidental actuel, que reste-t-il du Plaute original ? En un mot, nous revenons à la sacro-sainte question en traduction : qu'en est-il de la fidélité ?

\section{De la domestication au ludus : I'éthique du traduire par la fidélité au jeu}

Comme le rappelle P. Casanova dans un ouvrage récent consacré au phénomène de la " guerre des langues », la traduction occidentale, depuis l'Empire romain, est marquée par une tradition de domestication, c'est-à-dire un processus d'assimilation d'un texte étranger à sa propre culture ${ }^{26}$. Élément de domination à la fois linguistique, culturelle et politique, la traduction en Occident participerait donc, via une idéologie ethnocentrique sous-jacente, à effacer la présence de l'Autre perçu comme étranger. Or, grâce à l'influence au XIX ${ }^{\mathrm{e}}$ siècle de la philologie allemande qui redécouvre, après les humanistes, les textes anciens en langue originale, et grâce à l'instauration progressive dans le champ académique des Translation Studies en tant que discipline à part entière, le $\mathrm{xx}^{\mathrm{e}}$ siècle favorise la défense du courant inverse, jugé plus éthique, celui de la foreignization. Alors que la domestication revient à naturaliser ou annexer un texte - aucune métaphore coloniale n'est épargnée -, la foreignization, défendue en France par A. Berman, conserve les éléments d'étrangeté, linguistique et culturelle, du texte original ${ }^{27}$. Cette manière de traduire, qu'on appelle également traduction sourcière par opposition à une traduction cibliste, en revenant à la littéralité du texte, serait la seule façon d'être fidèle, et par là, éthique. Si aujourd'hui, ce débat binaire

25 Sur l'idée de la Grèce imaginaire dans la palliata, cf. M. Faure-ribreau, Pour la beauté du jeu : la construction des personnages dans la comédie romaine (Plaute, Térence), Paris, les Belles Lettres, 2012, p. 161.

26 P. Casanova, La langue mondiale : traduction et domination, Paris, Seuil, 2015.

27 A. Berman, L'Épreuve de l'étranger. Culture et traduction dans l'Allemagne romantique, Paris, Gallimard, 1984. 
s'essouffle un peu dans le champ académique, L. Venuti continue de critiquer cette tendance en révélant les pratiques éditoriales américaines qui privilégient dans leurs traductions un confort de lecture pour le public de destination ${ }^{28}$. Car favoriser l'étrangeté du texte original demanderait une lecture plus exigeante et attentive de la part du destinataire, qui devrait accepter soit de ne pas tout comprendre soit d'enrichir sa réception par des renseignements extérieurs. Dénoncer comme P. Casanova ou L. Venuti cette tendance occidentale à la traduction ethnocentrée, symptomatique d'une domination économique, littéraire et culturelle de la part de langues de traduction perçues comme supérieures (l'anglais en tête) est un geste bénéfique et nécessaire qui permet aux traducteurs d'avoir conscience des enjeux importants de leur travail. Mais pour autant, est-ce que toute traduction, quels que soient le texte, l'époque et le genre, doit exclure la domestication pour viser à l'étrangeté comme seule forme de fidélité ? Dans le cas d'un roman, lorsque le lecteur se trouve face à un texte appartenant à une culture radicalement différente de la sienne, il a tout loisir de revenir sur une phrase complexe, de consulter un dictionnaire ou de s'appuyer sur les notes du traducteur. Cela est possible parce que le genre romanesque s'accommode d'une lecture différée, inscrite dans un temps long fait d'allers-retours. Or, comment une pièce de théâtre, conçue pour la scène, peut-elle s'adapter à ce modèle textuel alors qu'elle repose sur la réception immédiate d'une œuvre éphémère se produisant dans un temps court et linéaire? Dans le cas d'une traduction fidèle de Plaute, une référence culturelle pointue, une plaisanterie linguistique rendue littéralement ne seront peut-être pas reçues par le public qui assiste à une représentation. Si l'effet est ponctuel, le dommage sera minime. Mais si une appréciation limitée se produit à chaque réplique, ce qui est voué à être le cas étant donné la densité de ses comédies, est-ce qu'une traduction littérale reposant sur un modèle textuel est vraiment une lecture fidèle et éthique de Plaute?

Dans une analyse d'anciennes traductions de Shakespeare, J.-M. Déprats, célèbre traducteur français aux éditions de la Pléiade, commente la difficulté de se trouver face à des termes désuets ou rares : « [...] même s'ils sont exacts, ils n'ont pas d'impact immédiat, ne sont pas immédiatement déchiffrables par l'oreille et sont donc, au théâtre, des mots morts ${ }^{29}$. »Un traducteur de théâtre ne travaille pas une matière textuelle qui restera figée sur la page, mais une matière orale qui va être prononcée sur scène. Lorsqu'on regarde une traduction savante du Miles gloriosus comme l'est la traduction universitaire d'A. Ernout, cette réalité

L. Venuti, The Translator's Invisibility: A History of Translation, London, New York, Routledge, 1995.

29 J.-M. DÉprats, “ Analyse comparative de plusieurs traductions françaises de Roméo et Juliette » in N. Vigouroux-Frey (éd.), Traduire le théâtre aujourd'hui ?, Rennes, Presses universitaires de Rennes, 1993, p. 91-101, p. 95. 
des “ mots morts » règne partout, jusque dans le titre. Si l'on prend pour cible un public adolescent $\mathrm{du} \mathrm{xxI}^{\mathrm{e}}$ siècle, quelle est la probabilité que certains ne comprennent pas même le titre Soldatfanfaron ${ }^{30}$ ? Cet adjectif soutenu, littéraire, loin du réalisme d'une conversation orale moderne, a besoin d'être actualisé. Et ce souci de la jouabilité du texte (performability dans les études anglosaxonnes) ne concerne pas seulement les préoccupations linguistiques de registre ou de prononciation : nous pouvons l'étendre au problème des références antiques qui ne peuvent exister sur scène accompagnées de notes de bas de page. Au théâtre, la volonté louable d'un traducteur de respecter l'étrangeté et la littéralité du texte peut ainsi conduire à l'incompréhension globale, ce qui nuirait au jeu. Et dans le cas de Plaute, il ne s'agit pas seulement du jeu théâtral, mais également du jeu comique. Prenons un procédé comique dans l'écriture de Plaute : l'onomastique. En effet, dans ses pièces, les personnages ont des noms signifiants qui renvoient aux différents rôles-types de la comédie ${ }^{31}$. Par exemple, Periplecomenes, un personnage du Miles dont il est question dans l'extrait que nous avons cité, est formé sur le verbe grec periplekô signifiant embrasser, enlacer. Par antonomase, le nom crée ainsi un décalage comique avec le personnage du vieux célibataire misogyne. Or, la traduction littérale empêche le spectateur d'apprécier cette plaisanterie qui participe à la construction cohérente de l'écriture théâtrale plautinienne. De même, les références culturelles sont inscrites dans le jeu comique : rarement gratuites, elles sont l'occasion de provoquer le rire, souvent aux dépens d'un autre personnage (comme l'utilisation de stéréotypes ethniques). Tout le système du monde antique n'étant plus accessible à la majorité, lorsqu'entre en jeu la question de l'humour, «l'adaptation au potentiel de la langue cible, mais aussi à la nature de son public et à son patrimoine culturel » est un principe traductologique non seulement raisonnable mais qui nous semble nécessaire. En modernisant la traduction de Plaute, nous défendons la « nature intrinsèquement cibliste des jeux de mots ", et plus largement, la nature intrinsèquement cibliste du genre théâtral de la comédie ${ }^{32}$. Mais en revendiquant une stratégie d'adaptation, nous ne pensons pas en revenir pour autant à une domestication comprise comme idéologie de domination culturelle. Au-delà de la binarité du débat qui oppose les sourciers aux ciblistes, nous ne pensons pas nous éloigner

30 Nous en avons fait l'expérience auprès de plusieurs classes de collégiens à Marseille et à Arles, dans le cadre du réseau « Experimentarium » qui organise des rencontres entre jeunes chercheurs et grand public.

31 M. Faure-ribreau, Pour la beauté du jeu. Sur les rôles-types et le répertoire de jeu, voir le deuxième chapitre: “Galerie de portraits. Les personae comiques chez Plaute et Térence », p. 66 sq.

32 F.Antoine et M. Wood (éd.), Traduire l'humour, Actes de la Journée d'étude d'ELEXTRA, du 5 mai 1997, Ateliers n. 15, Lille, Cahiers de la Maison de la Recherche, 1998, p. 27. 
de Plaute en favorisant son public, au contraire. En adaptant sa pièce à un langage et une réalité actuels, nous pourrions la faire redécouvrir au grand public. C'est cette démarche qui justifie le fait que ce geste moderne ne soit pas (seulement) une annexion ethnocentrique, mais aussi et surtout une forme de respect fidèle à l'auteur original, respect du principe qui gouverne toute son œuvre : le ludus.

Dans son ouvrage Aristote ou le vampire du théâtre occidental, F. Dupont théorise le fonctionnement de la comédie romaine grâce à la notion de “ ludisme », en l'opposant à la valeur mimétique du récit théâtral de la conception aristotélicienne :

On peut [...] regarder la comédie romaine comme un système cohérent organisé autour du jeu (ludus) de l'acteur, du public et de la performance rituelle, qu'on peut opposer au théâtre aristotélicien avec le récit (muthos), le poète, le texte et un lecteur qui n'intervient pas. Et si, dans la tragédie aristotélicienne, tout peut être ramené au muthos, dans la comédie romaine tout peut être ramené au ludus ${ }^{33}$.

Dans cette perspective, cela signifie que tous les éléments de vraisemblance qu'on pourrait chercher dans une pièce de théâtre d'apparence « réaliste » (c'est-àdire la cohérence de l'intrigue, les éléments référentiels à la société, la profondeur psychologique des personnages) n'existent pas chez Plaute : la comédie romaine ne relève pas de la fonction dramatique au sens aristotélicien du terme, mais seulement de la fonction ludique, c'est-à-dire de “ la gratuité du spectacle comique $^{34}$ ». Partant de ce postulat théorique, nous rejoignons la conclusion déjà établie de longue date par B.-A. Taladoire : " la loi essentielle du jeu comique [...] est beaucoup moins de faire vrai que de faire drôle ${ }^{35}$. „ L'idée d'une adaptation consiste donc à réactualiser cette valeur fondamentale du ludisme en défendant

33 F. Dupont, Aristote ou Le vampire du théâtre occidental, Paris, Flammarion, 2007, p. 230. Il faut bien distinguer l'actio, terme technique qui désigne le «jeu » des acteurs en tant que compétence professionnelle et le ludus, signifiant le « jeu » global, soit la valeur de divertissement théâtral, qui n'est pas le résultat de la seule actio, mais de toutes les conditions mises en œuvre par le rituel de la performance dans le cadre des Ludi (le script théâtral, l'attention respectueuse des spectateurs, etc.). Sur la sémantique du ludus, voir A. Nuti, Ludus e iocus. Percorsi di ludicità nella lingua latina, Rome, Viella, 1998.

34 M. Faure-ribreau, Pour la beauté du jeu, p. 355 : « Le terme ludus rend donc bien compte de la gratuité du spectacle comique [...] ». Dans son étude consacrée aux personnages de la palliata, l'auteur montre comment la codification artificielle du genre a pour but “ la beauté du jeu », c'est-à-dire l'exhibition de la machine théâtrale et non la mise en scène de situations réalistes.

35 B.-A. Taladoire, Essai sur le comique de Plaute, Monaco, Edition de l'Imprimerie Nationale de Monaco, 1956, p. 160. 
non pas la dimension référentielle du texte - soit le contenu littéral - mais la dimension comique des interactions verbales porteuses du jeu scénique. Car si l'on admet que, chez Plaute, les références à la société réelle sont toujours secondes par rapport à la primauté du jeu, le choix de privilégier en traduction la littéralité du texte plutôt que son potentiel comique pour la scène serait une erreur. Par conséquent, nous revendiquons par le biais d'une adaptation contemporaine du Miles la fidélité au ludus, faithfulness to the playfulness pour reprendre un principe défendu par D. Herskovits et D. Langworthy dans leur mise en scène du dramaturge allemand $\mathrm{H}$. von Kleist ${ }^{36}$. Le ludisme plautinien peut guider ainsi, en la légitimant, une traduction orientée vers l'efficacité comique du texte et de sa représentation. C'est ce principe-là de fidélité qui nous permettrait de rechercher une actualisation du style et des références, actualisation calibrée par rapport à notre public de destination. Car nous jugeons que le fait de modifier le contenu d'une référence est un changement secondaire par rapport à l'effet global de la pièce : établir, à travers une plaisanterie, une complicité de jeu avec le public. Que la blague concerne l'Iliade ou Game of Thrones, cela est seulement dû à un ajustement culturel nécessaire pour transcender la distance qui nous a séparés de Plaute.

Nous reprenons ici la conclusion établie par B. Métais-Chastanier et A. Carré à la suite d'entretiens en compagnie de plusieurs traducteurs qui travaillent pour la scène, notamment I. Bonnaud et M. Bastin-Hammou, spécialistes des tragiques grecs :

Le cas du théâtre antique exemplifie de manière prégnante une réalité que les traducteurs de théâtre contemporain connaissent également : celle du contexte de réception et la façon dont il modifie les effets du texte, allant jusqu'à obscurcir le sens de certaines séquences dont le saisissement initial était pourtant immédiat ${ }^{37}$.

Cela ne laisse souvent qu'une alternative : soit le traducteur considère le texte comme un objet textuel, historiquement situé, visant une littéralité sans faille, quitte à n'être appréhendable que par un lectorat de spécialistes ; soit il le traite comme une matière littéraire vivante, universelle, destinée à la scène, et privilégie la voie de l'adaptation. Et dans le cas de la comédie plautinienne, une traduction pensée en vue d'une représentation peut difficilement choisir l'exactitude historique sans perdre la notion de jeu. J.-M. Déprats le répète à propos de la traduction de Shakespeare :

36 D. Herskovits et D. Langworthy, “Faithfulness to the Playfulness », Theater, 341 , (2004), p. 61-65.

37 B. Métais-Chastanier et A. Carré, “ Où commence la dramaturgie ? », Traduire, 223 : “Tribunal et théâtre, faites entrer le traducteur », (déc. 2010), p. 89-101. 
Une traduction qui n'est pas jouable comprend mal la nature du texte [...] et sa destination. Elle peut être exacte, inventive, écrite dans une belle langue, mais si elle ne permet pas la pratique théâtrale, elle reste essentiellement infidèle ${ }^{38}$.

C'est pourquoi, dans la perspective de la fidélité au ludus, à la fois jeu théâtral et jeu comique, nous défendons le fait d'adapter aux codes contemporains une pièce comme le Miles gloriosus. Une objection possible à une telle entreprise reste le fait qu'une traduction située dans un contexte générationnel (expressions du parler « jeune », références culturelles du temps présent) court le risque de vieillir prématurément. Nous répondrons que l'éphémère n’est pas nécessairement une faiblesse. Au contraire, nous pensons que c'est une caractéristique attachée au théâtre en général, et à Plaute en particulier, dont les textes étaient conçus comme des playscripts renouvelables. Comme l'explique A. Arcellaschi à propos du Miles : “chaque représentation s'est chargée de nouvelles allusions à l'actualité contemporaine ». Il définit la mise en scène comme “l'occasion d'une recréation, et de rajouts qui se veulent réajustements à l'actualité39. „ Une adaptation contemporaine intitulée Le militaire Kislapet peut alors se concevoir, sous l'autorité de Plaute, comme un nouveau réajustement provisoire à l'actualité.

\author{
Clara Daniel \\ Doctorante en littérature générale \\ et comparée \\ Université Aix-Marseille \\ CNRS, TDMAM, CIELAM \\ Aix-en-Provence, France \\ clarajuliedaniel@gmail.com
}

\footnotetext{
38 J.-M. DÉprats, “ Traduire Shakespeare pour le théâtre? » in P. Bensimon (éd.), “ Traduire le dialogue, Traduire les textes de théâtre », Palimpsestes, vol. 1, 1987, p. 55.

39 A. Arcellaschi, “ La composition du Miles gloriosus », REL 71, (1993), p. 44- 54.
} 